\title{
Circumferential Spinal Cord Decompression through a Single Posterior Approach with Microendoscopy for Thoracic and Thoracolumbar Ossification of the Posterior Longitudinal Ligament
}

\author{
Shoji Seki, Hayato Mine, Yoshiharu Kawaguchi, Hiroto Makino, Tomoatsu Kimura \\ Department of Orthopaedic Surgery, Faculty of Medicine, University of Toyama, Toyama, Japan
}

\begin{abstract}
Thoracic and thoracolumbar ossification of the posterior longitudinal ligament (OPLL) can be difficult to treat due to the anatomical position. The purpose of this study was to report the significance of a novel surgical technique that represented two cases of thoracic or thoracolumbar OPLL. The first patient was a 72-year-old woman who had a beak-type OPLL at the T11/12. The second was a 45-yearold woman who had a beak-type OPLL at the T12/L1. We performed circumferential spinal cord decompression through a single posterior approach with microendoscopy in both cases. The postoperative computed tomography revealed the complete removal of the OPLL, and the magnetic resonance imaging confirmed adequate decompression of the spinal cord. Preoperative symptoms were substantially improved in both patients. To date, we have used this novel technique to treat five patients with thoracic or thoracolumbar OPLL. This new surgical technique is likely to be useful in patients with a beak-type OPLL of the thoracic or thoracolumbar spine.
\end{abstract}

Keywords: Ossification of the posterior longitudinal ligament of the spine; Spinal fusion; Decompression; Spinal cord; Dura mater

\section{Introduction}

Ossification of the posterior longitudinal ligament (OPLL) occurs frequently in Asian people. The incidence of thoracic OPLL in Japanese is about $0.8 \%[1,2]$.

There are two operative approaches, the anterior and posterior approaches, for thoracic and thoracolumbar OPLL. The anterior approach requires thoracotomy, sternal longitudinal splitting, or a transpleural and extrapleural approach, imposing substantial invasive stress on patients and frequently results in cerebrospinal fluid leakage as a complication [3-5]. In addition, the anterior approach requires deep access to the vertebra, making it extremely difficult, in many cases, to remove the ossified lesions, and is associated with a greater risk of respiratory complications, injuries to the dura mater, and cerebrospinal fluid leakage compared with the posterior approach $[3,6]$. In contrast, although decompression for OPLL via a posterior approach is relatively simple in terms of technique and the management of complications, it does not

Received Nov 14, 2014; Accepted Dec 3, 2014

Corresponding author: Shoji Seki

Department of Orthopaedic Surgery, Faculty of Medicine, University of Toyama,

2630 Sugitani, Toyama 930-0194, Japan

Tel: +81-76-434-7353, Fax: +81-76-434-5035, E-mail: seki@med.u-toyama.ac.jp 
provide satisfactory visibility on the ventral side of the dura mater and is often performed in combination with anterior decompression [1,7]. In thoracic OPLL, since the ossification is located anteriorly, the presence of a thoracic kyphosis means that using only posterior decompression may, sometimes, be inadequate [1]. The decompression of an ossified lesion positioned anteriorly through a single posterior approach is technically demanding and may result in postoperative neurological deterioration due to the excessive traction of the dura mater or a blinded resection of ossified lesions [8].

Here, we described a method for circumferential spinal cord decompression through a single posterior approach with microendoscopy. To date, we have used this novel technique to treat five patients with thoracic OPLL. We showed this procedure in the treatment of two representative patients with thoracic or thoracolumbar OPLL.

\section{Technical Note}

\section{First OPLL case}

1) History and neurological findings

A 72-year-old woman had undergone cervical laminoplasty for cervical OPLL. She had extremely strong pain from the lower back to the hip area through the anterior region of the thigh, both in walking and at rest. Walking was also difficult because of the weakened muscles of the lower extremities. The neurological examination revealed muscle weakness in the bilateral iliopsoas muscles, quadriceps femoris, tibialis anterior, and gastrocnemius as well as mild hypalgesia of the right lower extremity. The distribution of pain, motor weakness, and tendon hyperreflexia suggested thoracic myelopathy.

\section{2) Imaging findings}

A plain lateral X-ray and sagittal view computed tomography (CT) revealed a cervical OPLL and a beak-type OPLL at the T11/12 level, which occupied $60 \%$ of the spinal canal (Fig. 1). A magnetic resonance imaging also revealed that the spinal cord at this level was strongly compressed from the front and made flat (Fig. 2). On the basis of these findings, thoracic myelopathy caused by thoracic OPLL
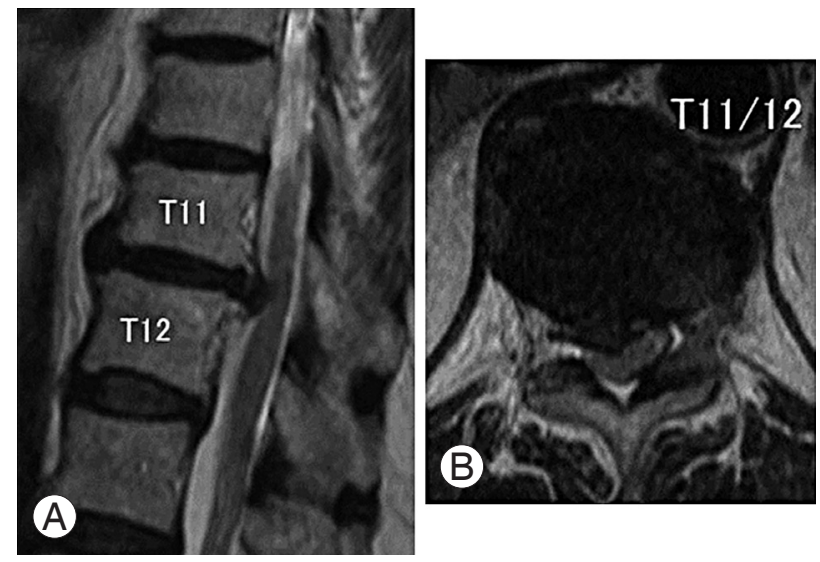

Fig. 2. Preoperative magnetic resonance imaging. (A) Sagittal view, (B) axial view.
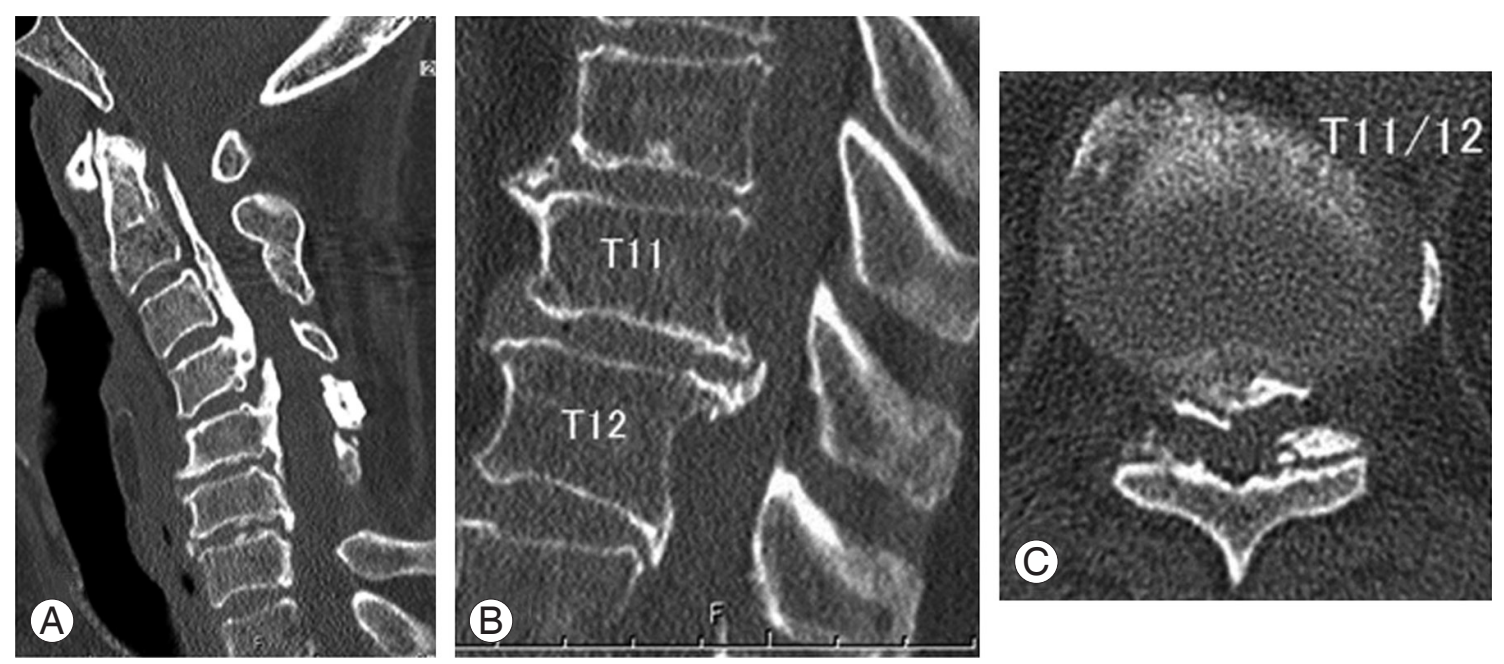

Fig. 1. Preoperative computed tomography scan. (A) Sagittal view of the cervical spine. The patient underwent cervical laminoplasty for the ossification of the posterior longitudinal ligament. (B) Sagittal view of the thoracic spine. (C) Axial view of the thoracic spine. 
was diagnosed.

\section{3) Operation}

The operative technique was planned to combine posterior decompression and fusion through a single posterior approach with microendoscopic resection of the anterior OPLL (Fig. 3). After a posterior midline incision was performed and the paravertebral muscles were exposed, pedicle screws were inserted into the upper and lower vertebrae of the affected intervertebral disc, using the free hand technique, followed by laminectomy and bilateral facetectomy to release the dura mater and nerve roots (Fig. $3 \mathrm{~A})$. After the bilateral roots were preserved with a micro spurtle, bleeding from the epidural venous plexus was stopped with a bipolar electrical scalpel. The intervertebral disc was removed bilaterally, and the OPLL that was positioned anteriorly to the dura mater was recognized (Fig. 3B). The ossified lesion on either side of the dura mater was resected under direct vision (Fig. 3C). Since adhesion of the dura mater and OPLL was noted, the dura mater was carefully detached from the OPLL with a knife or spurtle under the microendoscope that was inserted through the intervertebral disc space (Fig. 4A-C). The ossified lesion that was ventral to the dura mater was bilaterally excised with an air drill under the microendo- scope, during which the dura mater was protected with particular care (Figs. 3D, E, 4D, E). We also took particular care to make sure that the displacement of the dura on retraction was less than one-third of the width of the dura mater. The microendoscope was then inserted from the opposite side of the intervertebral disc space, and the ossified mass that remained in the median area was excised under visual observation (Figs. 3E, 4F-H). This bilateral OPLL could be removed without retraction of the dura mater because the 25-degree oblique lens of the microendoscope provided good visibility of the ventral side of the dura mater. Upon completion of the decompression, the microendoscope was inserted from both sides of the intervertebral disc space to confirm complete circumferential decompression of the spinal cord (Figs. 3F, G, 4I-L). Bleeding from the vertebral bodies was controlled by filling defects with hemostatic gelatin sponge or application of a hemostatic bone wax under microendoscopy. Fig. 5 shows an intraoperative image and postoperative $\mathrm{X}$-ray after circumferential spinal cord decompression and posterior fixation.

\section{4) Postoperative course}

The postoperative CT revealed the complete removal of the OPLL, and the magnetic resonance imaging con-
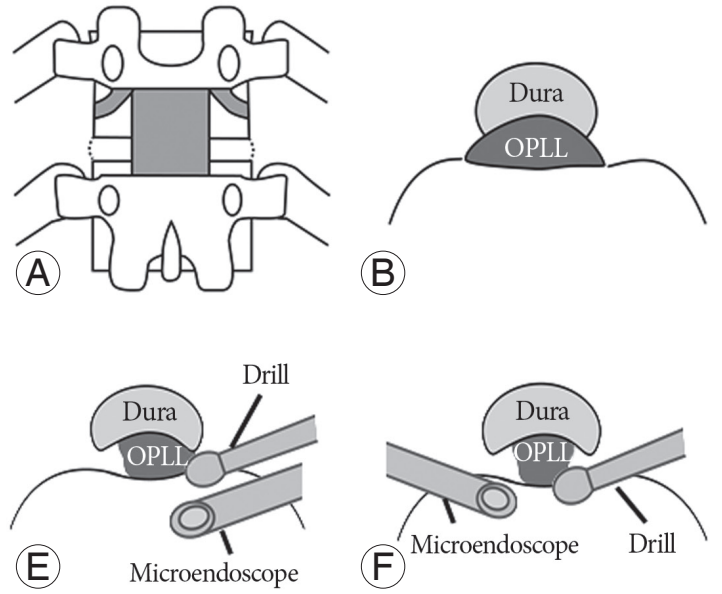

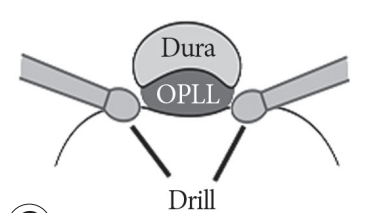

(C)

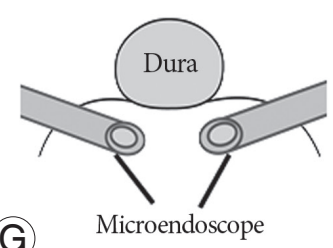

Fig. 3. Circumferential spinal cord decompression with microendoscopic surgery. (A) A posterior view of the spine. Laminectomy and facetectomy were achieved after pedicle screw insertion. (B) An axial views of the spine. The intervertebral disc was removed bilaterally from around the dura mater. (C) An axial views of the spine. Ossification of the posterior longitudinal ligament (OPLL) positioned on the lateral aspects of the dura was removed with an air drill under direct observation. If adhesion between the dura mater and OPLL was recognized, the dura was carefully detached from the OPLL with a micro spurtle under microendoscopy. (D, E) An axial views of the spine. Microendoscopic resection of OPLL occurring ventrally to the spinal cord is performed bilaterally to the dura mater. (F) An axial views of the spine. Remaining OPLL around the central and ventral dura mater is removed with the air drill under observation by microendoscopy from the opposite side. (G) An axial views of the spine. Circumferential spinal cord decompression is confirmed by microendoscopy from both sides. 

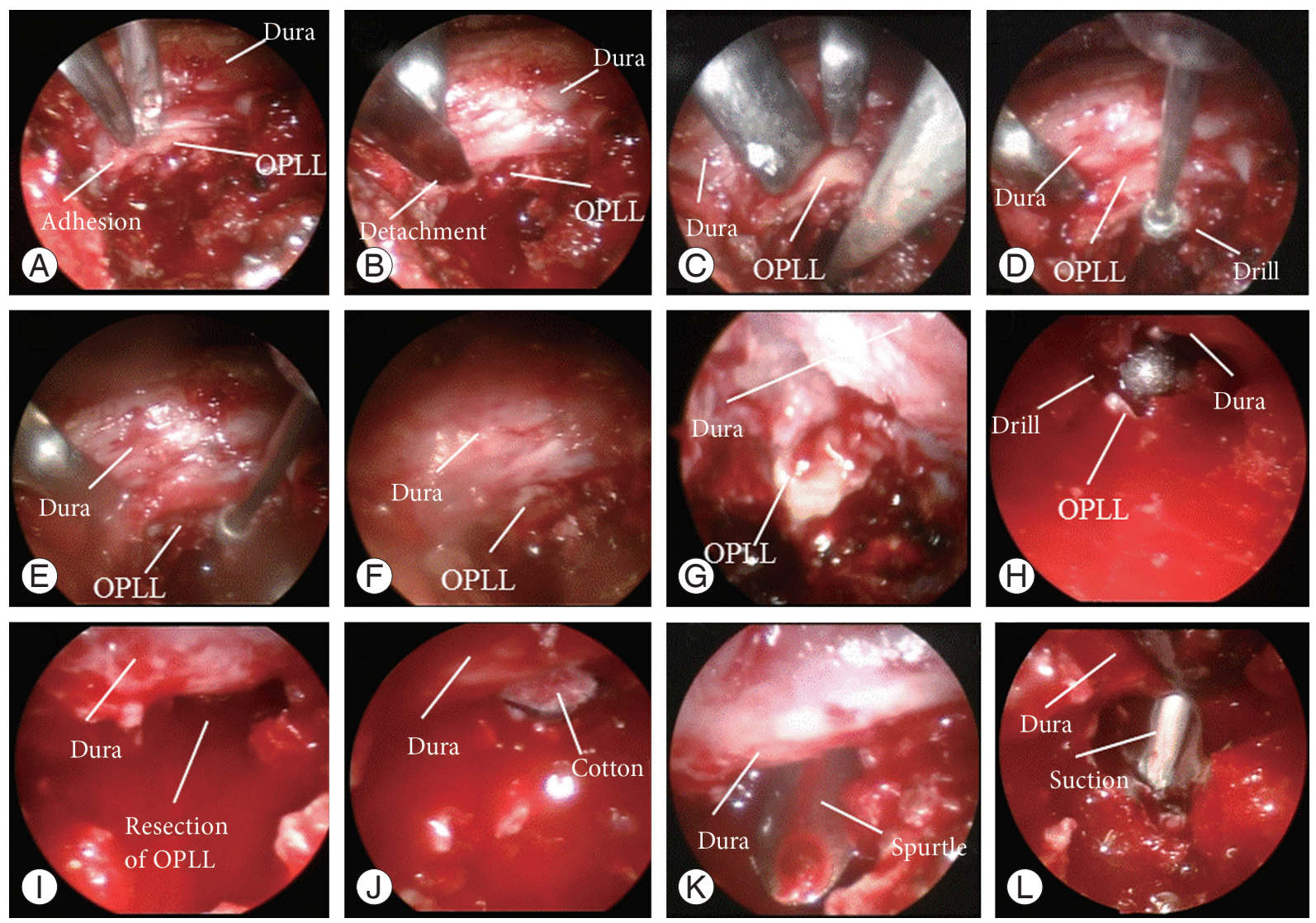

Fig. 4. Intraoperative microendoscopic image. (A) Adhesion is recognized between the dura and ossification of the posterior longitudinal ligament (OPLL). (B) The adhesion is detached with a knife. (C) OPLL that is positioned ventral to the dura mater is recognized. (D) OPLL of the ventral dura is shaved with the air drill. (E) OPLL is excised with the air drill, toward the ventral side of the dura. (F) The photo shows the remaining OPLL. (G) The microendoscope is inserted from the opposite side. The remaining OPLL is recognized. (H) The OPLL of the ventral dura is shaved with the air drill. The microendoscope is inserted from the opposite side. (I, J) Decompression of the spinal cord is performed. The OPLL is completely removed. The surgical cotton is visible on the opposite side. (K, L) The microendoscope is inserted from the opposite side and decompression of the spinal cord is confirmed. The micro spurtle and suction tube are visible on the opposite side.
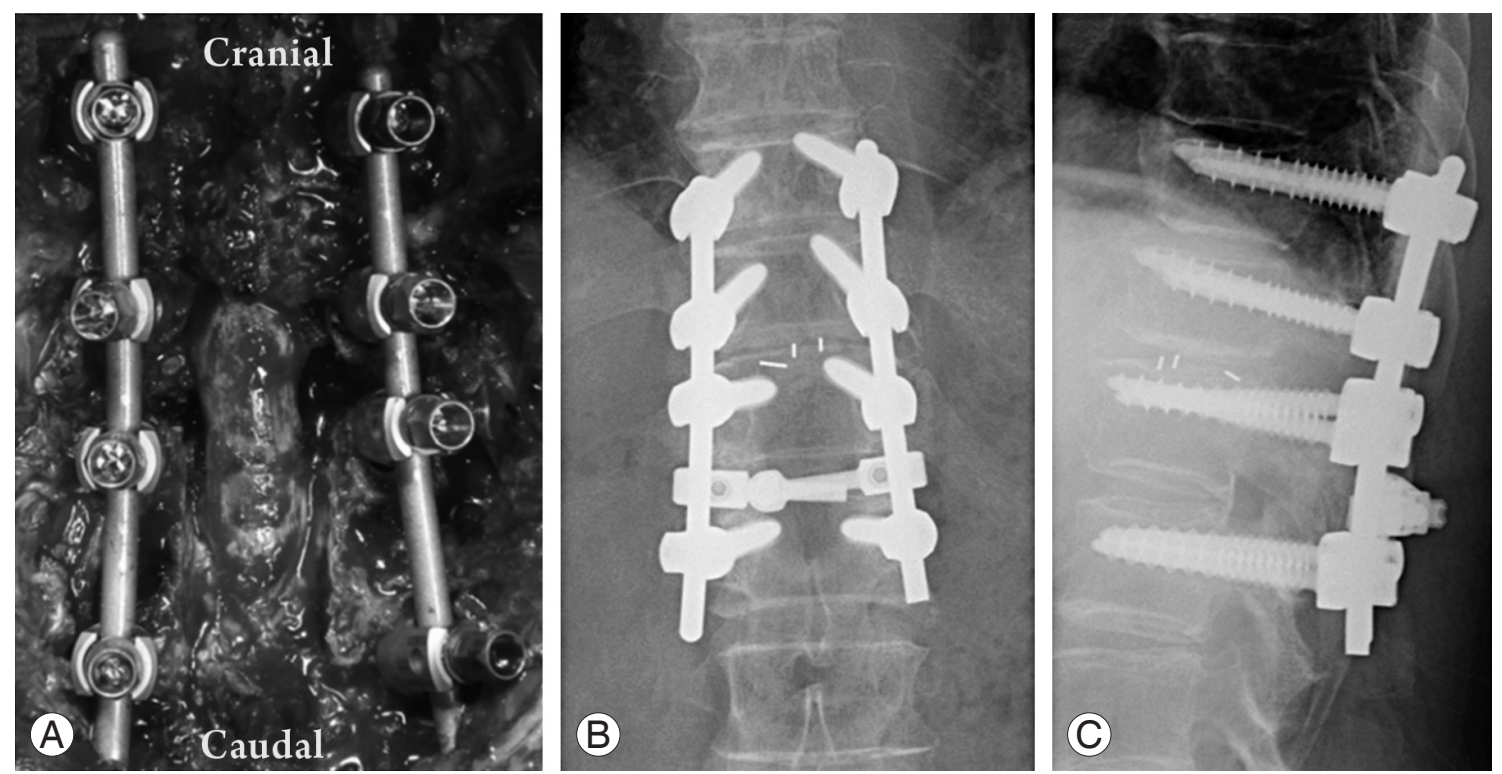

Fig. 5. Intraoperative image and postoperative X-ray. (A) Intraoperative image, (B) frontal view, (C) sagittal view. 
firmed adequate decompression of the spinal cord (Figs. 6, 7). Her pain was substantially improved and she became able to walk.

\section{Second OPLL case}

A 45-year-old woman had extremely strong pain from the lower back to the hip area through the anterior region of
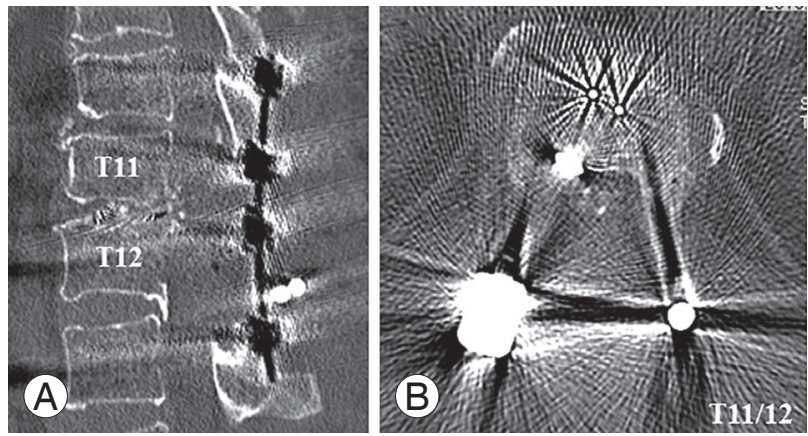

Fig. 6. Postoperative computed tomography scan. The ossification of the posterior longitudinal ligament is completely removed. (A) Sagittal view, (B) axial view.
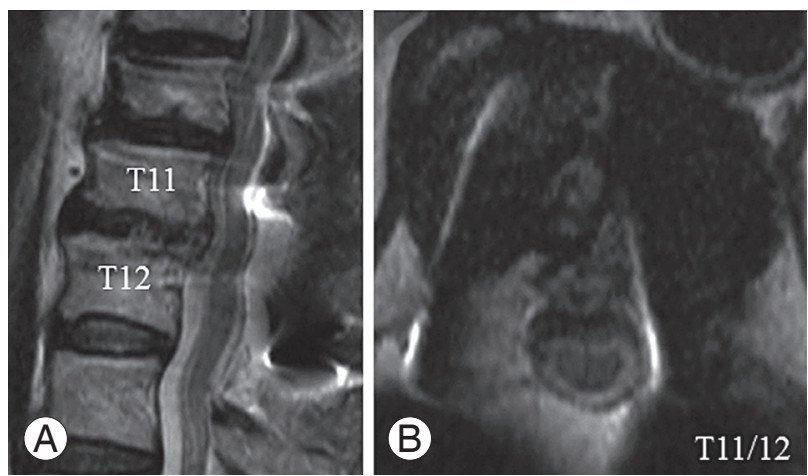

Fig. 7. Postoperative magnetic resonance imaging. Decompression of the spinal cord is confirmed. (A) Sagittal view, (B) axial view. the thigh both while walking. She also had difficulty in walking because of the leg pain. The distribution of the pain area and motor weakness suggested disturbance at the thoracolumbar level. A sagittal CT scan revealed a beak-type OPLL at the T12/L1 level (Fig. 8A, B). The surgery was performed by the same procedure as described above. The displacement of the dura on traction was less than one-third of the width of dura mater. The postoperative CT showed the complete removal of the OPLL (Fig. $8 \mathrm{C}, \mathrm{D})$. Both muscle strength and hypalgesia of the lower extremities returned to near-normal. Her pain was substantially improved and she became able to walk.

\section{Discussion}

Microendoscopic discectomy for lumbar disc herniation was first reported by Foley and Smith [9] in 1997 [10]. This methodology has recently been applied to a variety of diseases, including extraforaminal stenosis of the lumbar spine [11], lumbar spinal stenosis [12], and cervical myelopathy [13], among others. However, these operative procedures have a learning curve [14]. In this study, we used the lumbar microendoscope to resect the thoracic and thoracolumbar OPLL. We routinely used this microendoscopic system for surgical treatment of lumbar disc herniation and lumbar spinal stenosis, and we performed the presented procedures without difficulty. To our knowledge, this is the first report of decompression of an OPLL positioned ventral to the spinal cord with a microendoscope.

Ultrasonography [15-17] and navigation [16] have been used to confirm spinal cord decompression after surgical treatment for OPLL and intervertebral disc herniation. Ultrasonography is useful in confirming decompression,
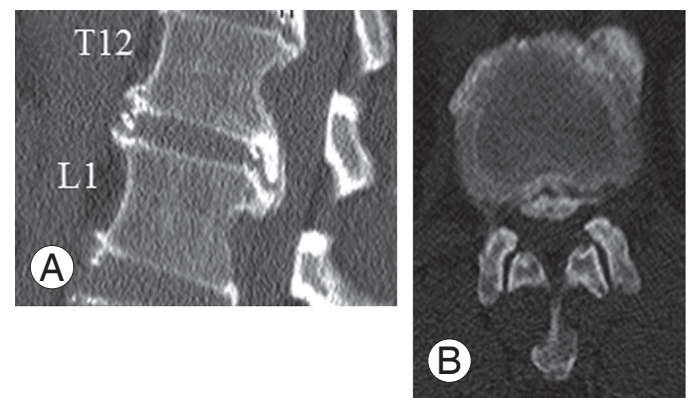
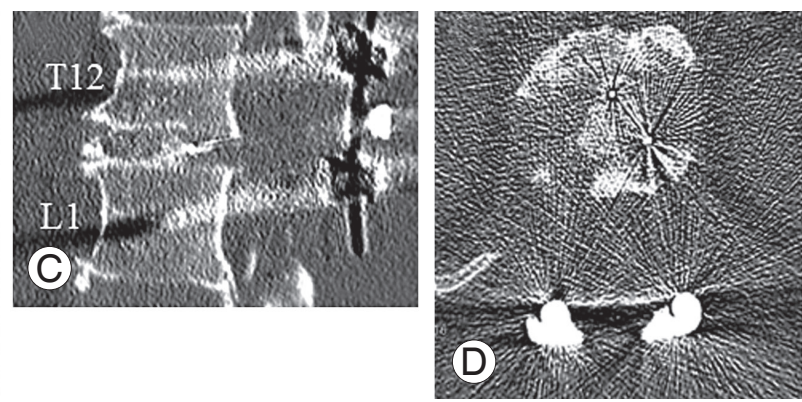

Fig. 8. Preoperative and postoperative computed tomography (CT) scan. A beak-type ossification of the posterior longitudinal ligament is positioned at the T12/L1 level. (A) Sagittal view of preoperative CT. (B) Axial view of preoperative CT. (C) Sagittal view of postoperative CT. (D) Axial view of postoperative CT. 
although simultaneous decompression and confirmation of spinal cord decompression with this method are difficult. In contrast, microendoscopy has the advantage in that microendoscopic decompression is performed under simultaneous confirmation of spinal cord decompression. Furthermore, the 25-degree oblique lens of the microendoscope provides good visibility of the ventral side of the dura mater (Fig. 4I-L).

A multi-institutional study of thoracic OPLL reported a mean recovery rate in the Japanese Orthopaedic Association score of $36.8 \%$, after the implementation of various surgical procedures. This ratio did not significantly differ among operative procedures, but it tended to be higher with procedures that provided circumferential spinal cord decompression [18]. A second study reported that using only posterior decompression is inadequate in thoracic OPLL, since the ossified masses are located anteriorly and are due to thoracic kyphosis [1], while a third study reported that the resection of an ossified lesion that was positioned ventral to the spinal cord through a single posterior approach was technically demanding and might result in postoperative neurological deterioration due to the excessive traction of the dura mater or inadvertent resection of ossified lesions [8]. In light of these points, our operative procedure was reasonable since it enabled decompression with simultaneous microendoscopic confirmation of the targeted ossification.

For the presented patients, myelopathy occurred in the lower part of the thoracic spine and was localized to a single intervertebral disc, for which either an anterior or a posterior approach could have been selected. Because the spinal cord was severely compressed, however, we considered that using only posterior decompression and fusion was inadequate and judged that resection of the ossified mass was necessary. We therefore selected circumferential spinal cord decompression through a single posterior approach. As described above, this operative technique provided several challenges, namely securing the visibility of the ventral side of the dura mater and confirming the decompression of the spinal cord. We used a microendoscope to resolve these issues. The major advantage of microendoscopy is that it provides adequate visibility on the ventral side of the dura mater even when a posterior approach is made, since it has an oblique lens. The disadvantage of combined microendoscopy is poor visibility when bleeding is massive, and microendoscopy may not be useful in patients for whom bleeding is difficult to control or who require extensive resection of ossification, which will probably lead to massive bleeding. The combined use of microendoscopy needs to be carefully examined before surgery.

\section{Conflict of Interest}

No potential conflict of interest relevant to this article was reported.

\section{References}

1. Kurosa Y, Yamaura I, Nakai O, Shinomiya K. Selecting a surgical method for thoracic myelopathy caused by ossification of the posterior longitudinal ligament. Spine (Phila Pa 1976) 1996;21:1458-66.

2. Tsuyama N. Ossification of the posterior longitudinal ligament of the spine. Clin Orthop Relat Res 1984; (184):71-84.

3. Fujimura Y, Nishi Y, Nakamura M, Toyama Y, Suzuki N. Long-term follow-up study of anterior decompression and fusion for thoracic myelopathy resulting from ossification of the posterior longitudinal ligament. Spine (Phila Pa 1976) 1997;22:305-11.

4. Ido K, Shimizu K, Nakayama Y, et al. Anterior decompression and fusion for ossification of posterior longitudinal ligament in the thoracic spine. J Spinal Disord 1995;8:317-23.

5. Kawahara N, Demura S, Murakami H, Tomita K. Transvertebral herniotomy for T2/3 disc herniation: a case report. J Spinal Disord Tech 2009;22:62-6.

6. Min JH, Jang JS, Lee SH. Clinical results of ossification of the posterior longitudinal ligament (OPLL) of the thoracic spine treated by anterior decompression. J Spinal Disord Tech 2008;21:116-9.

7. Tomita K, Kawahara N, Baba H, Kikuchi Y, Nishimura H. Circumspinal decompression for thoracic myelopathy due to combined ossification of the posterior longitudinal ligament and ligamentum flavum. Spine (Phila Pa 1976) 1990;15:1114-20.

8. Takahata M, Ito M, Abumi K, Kotani Y, Sudo H, Minami A. Clinical results and complications of circumferential spinal cord decompression through a single posterior approach for thoracic myelopathy caused by ossification of posterior longitudinal ligament. Spine (Phila Pa 1976) 2008;33:1199-208.

9. Foley KT, Smith MM. Microendoscopic discectomy. 
Tech Neurosurg 1997;3:301-7.

10. Perez-Cruet MJ, Foley KT, Isaacs RE, et al. Microendoscopic lumbar discectomy: technical note. Neurosurgery 2002;51(5 Suppl):S129-36.

11. Matsumoto M, Chiba K, Ishii K, Watanabe K, Nakamura M, Toyama Y. Microendoscopic partial resection of the sacral ala to relieve extraforaminal entrapment of the L-5 spinal nerve at the lumbosacral tunnel: technical note. J Neurosurg Spine 2006;4:342-6.

12. Khoo LT, Fessler RG. Microendoscopic decompressive laminotomy for the treatment of lumbar stenosis. Neurosurgery 2002;51(5 Suppl):S146-54.

13. Minamide A, Yoshida M, Yamada H, et al. Clinical outcomes of microendoscopic decompression surgery for cervical myelopathy. Eur Spine J 2010;19:487-93.

14. Nowitzke AM. Assessment of the learning curve for lumbar microendoscopic discectomy. Neurosurgery 2005;56:755-62.

15. Seichi A, Chikuda H, Kimura A, et al. Intraoperative ultrasonographic evaluation of posterior decompression via laminoplasty in patients with cervical ossification of the posterior longitudinal ligament: correlation with 2-year follow-up results. J Neurosurg Spine 2010;13:47-51.

16. Tian W, Weng C, Liu B, et al. Intraoperative 3-dimensional navigation and ultrasonography during posterior decompression with instrumented fusion for ossification of the posterior longitudinal ligament in the thoracic spine. J Spinal Disord Tech 2013;26: E227-34.

17. Tokuhashi Y, Matsuzaki H, Oda H, Uei H. Effectiveness of posterior decompression for patients with ossification of the posterior longitudinal ligament in the thoracic spine: usefulness of the ossification-kyphosis angle on MRI. Spine (Phila Pa 1976) 2006;31:E26-30.

18. Matsumoto M, Chiba K, Toyama Y, et al. Surgical results and related factors for ossification of posterior longitudinal ligament of the thoracic spine: a multiinstitutional retrospective study. Spine (Phila $\mathrm{Pa}$ 1976) 2008;33:1034-41. 